\title{
Evolución De La Desigualdad Y Pobreza En Nicaragua En El Periodo De 1991 A 2014
}

\author{
Mc. Carlos Ernesto Luquez Gaitán \\ Mc. Ernest Yasser Núñez Betancourt
}

Doctorante División de Ciencias Económico Administrativas,

Universidad Autónoma Chapingo, Chapingo, México

\section{Dr. Manuel del Valle Sánchez}

Profesor-Investigador división de Ciencias Económico Administrativas,

Universidad Autónoma Chapingo, Chapingo, México

Doi:10.19044/esj.2018.v14n31p337 URL:http://dx.doi.org/10.19044/esj.2018.v14n31p337

\begin{abstract}
This paper discusses the situation of the evolution of inequality and poverty from a historical perspective, including the structural reforms in Nicaragua during the decade of 1990. It also examines the decline in poverty in Nicaragua from 1991 through World Bank indicators. Methods of measuring inequality and poverty such as the Gini coefficient, GDP per capita, and the incidence rate of poverty, as well as the poverty gap, are used. It includes the results of quantitative assessment of poverty and inequality, and concludes with a decline in poverty. There is evidence of a steady increase in real GDP and a steady trend in the unemployment rate.
\end{abstract}

Keywords: Inequality, poverty, Gini coefficient, GDP per capita, unemployment

\section{Resumen}

El artículo aborda la situación de la evolución de la desigualdad y la pobreza desde una perspectiva histórica, incluyendo las reformas estructurales en Nicaragua durante la década de 1990.También examina la disminución de la pobreza en Nicaragua desde 1991 a través de indicadores del Banco Mundial; para ello se utilizan los métodos de medición de desigualdad y pobreza como el coeficiente de Gini, el PIB per cápita y la tasa de incidencia de la pobreza, así como la brecha de pobreza. Este arículo incluye también los resultados de valoración cuantitativa de la pobreza y la desigualdad; concluyendo con una disminución de la pobreza que se evidencia en un continuo aumento del PIB real y una tendencia estable de la tasa de desempleo. 
Palabras clave: Desigualdad, Pobreza, Coeficiente de Gini, PIB per cápita, Desempleo.

\section{INTRODUCCIÓN}

Una de las maneras de describir algún fenómeno es proponer definiciones contrarias del problema en cuestión. En el caso de la desigualdad se podría ver desde el objetivo que es la igualdad; la igualdad como se cuestionó (Sen Amartya, 1992): "igualdad, ¿de qué? Podemos hablar de igualdad de status, étnica, de género, de renta, educativa, de riqueza, de salud, politica, etc. Se afirma que la idea de igualdad hace frente a dos formas de diversidad: la básica heterogeneidad humana y la multiplicidad de variables desde las que se puede juzgar la igualdad".

En este sentido, lo que intenta explicar es que dicha heterogeneidad humana lleva a una serie de disensiones en el valor y en el papel de la igualdad, sobre todo cuando se comparan variables diferentes. En otras palabras, el ser humano tiende a desviar su atención a variables categoriales diversas y desvirtúa la idea de igualdad, lo cual, aunque no sea intencional, no permite una búsqueda eficiente de la misma.

"La potente retórica de la igualdad del hombre a menudo suele desviar la atención de estas diferencias. Aunque tal retórica, por ejemplo, "todos los hombres nacen iguales", se ha considerado siempre como parte esencial del igualitarismo, las consecuencias de pasar por alto esas diferencias entre los individuos, de hecho, pueden llegar a ser muy poco igualitarias, al no tener en cuenta el hecho de que el considerar a todos por igual puede resultar en que se dé un trato desigual a aquellos que se encuentran en una posición desfavorable. Los requerimientos de igualdad substantiva pueden ser particularmente exigentes y complejos cuando hay que contrarrestar un grado importante de desigualdad previa (Sen Amartya, 1992)."

Desgraciadamente para muchos, y no desde hace poco tiempo, la historia de la humanidad no es la historia de la igualdad, sino de la desigualdad entre los hombres. Incluso parece que existe un acuerdo generalizado entre teóricos y científicos sociales sobre el hecho de que a lo largo de la historia de la humanidad, el desarrollo de las distintas civilizaciones y el progreso de las sociedades en general, está fundamentado en la diferencia y la desigualdad, ya sea ésta producto de la edad o el género, de la posesión de bienes tangibles o del control de los medios de producción.

¿Qué es lo que provoca la desigualdad?; la heterogénea distribución de la riqueza está intrínseca en el sistema de producción actual; es decir en el que el capitalismo crea desigualdades al generar la reproducción y concentración del capital, se aprecia que cada uno de los componentes dentro del sistema tiene intereses particulares, siendo este un factor muy importante para considerar el aspecto subjetivo de la desigualdad. 
Es importante analizar los factores que contribuyen a la desigualdad económica en Nicaragua. En primer lugar, se analizará desde el punto de vista teórico y luego se analizarán, a través de las estadísticas, el grado de pobreza y desigualdad y su evolución a partir de 1991, año en que se transformó la estructura económica de Nicaragua y comenzó a regir la corriente neoliberal. A partir de las estadísticas se concluirá el análisis partiendo siempre de la teoría económica.

En lo que respecta a la zona de estudio, el país tiene una superficie de 130,370 km2; es el país más grande de Centro América y la población censada del 2016 fue 6169,668 lo que da como resultado una densidad poblacional de $47.32 \mathrm{hab} / \mathrm{km}^{2}$.

El PIB per cápita para el 2015 fue de 2047.40 dólares americanos anuales, lo que ubica a Nicaragua como un país de ingreso medio-bajo según la clasificación que ofrece el Banco Mundial. De igual forma presentó un Índice de Desarrollo Humano de 0.631 "El Índice de Desarrollo Humano $(I D H)$ es un indicador creado por el Programa de las Naciones Unidas para el Desarrollo (PNUD) con el fin de determinar el nivel de desarrollo que tienen los países del mundo. Fue ideado con el objetivo de conocer, no sólo los ingresos económicos de las personas en un país, sino también para evaluar si el país aporta a sus ciudadanos un ambiente donde puedan desarrollar mejor o peor su proyecto y condiciones de vida (Pampillón, 2009); ubicando a Nicaragua como un país de desarrollo medio.

\section{MATERIALES Y MÉTODOS}

\section{Diferentes enfoques respecto a la Desigualdad.}

Para analizar la desigualdad se debe definir a qué se refiere concretamente la desigualdad: "es la disparidad fundamental que permite a una persona ciertas oportunidades materiales y se las niega a otra. Las medidas relativas a la desigual distribución de la renta permiten conocer cómo se produce el reparto del crecimiento y el desarrollo entre los distintos grupos sociales. Las distribuciones más equitativas de la renta son una de las características de las sociedades desarrolladas, mientras que las economías más atrasadas suelen tener un reparto de la renta y la riqueza más desigual (Ortiz, 2009).

La desigualdad se relaciona con la pobreza no como causante, sino más bien como un intensificador de la pobreza; es decir, en un mundo igual la pobreza sería un problema generalizado y sería una riqueza homogénea donde no habría punto de comparación sobre cual es más o menos pobre o más o menos rico. En otras palabras, la desigualdad genera medidas de referencia tomando en cuenta los niveles de ingreso más elevados y contrastándolos con otros. 
No toda la desigualdad es mala. Hay desigualdad mala, que es la que genera desánimo, y desigualdad buena, la que estimula y convence a la gente de que es posible perseguir y alcanzar la felicidad. También hay desigualdad justa e injusta; la primera, por quienes han triunfado a base de trabajo, esfuerzo e innovando en beneficio del mundo y la otra, propiciada por quienes se han hecho ricos por medios dudosos (Torres, 2017).

Es claro que para esta corriente las desigualdades sociales son una construcción socio-histórica, expresión de las formas específicas de estructuración de lo social que han gestado los seres humanos a lo largo de su historia y que impiden el reencuentro, en un nivel superior, de los fundamentos del igualitarismo. De esta manera, la tarea política prioritaria es la construcción de un nuevo orden social sustentado en la propiedad colectiva de los medios de producción y en la distribución socializada de la riqueza social. En esta perspectiva, el debate sobre la desigualdad social queda vinculado al tema de la distribución del producto social entre grupos de individuos estructuralmente conformados como clases sociales, en función de la posición que ocupan en el sistema productivo y la relación que guardan con respecto a los medios de producción. (Cortés)

Teniendo en cuenta lo anteriormente comentado por diferentes autores podemos observar que la desigualdad es un término que tiene mucho que ver con la distinción de clases sociales y discriminación desde muchas índoles. La desigualdad se evidencia desde una perspectiva multidimensional donde juegan un papel fundamental diferentes factores como la educación, las oportunidades de trabajo, el nivel económico, influencias políticas y desarrollo personal. Todos estos factores van a determinar que el ser humano tiene una posición más o menos favorable en nuestra sociedad actual.

Pobreza: La pobreza se debe de entender, para efecto de un correcto análisis de esta investigación, como la falta de riqueza para acceder a bienes y servicios necesarios para el desarrollo de las actividades necesarias para vivir por parte de un individuo al igual que la realización de todas las actividades que le permitan desarrollarse de manera adecuada.

Aunque no sea un concepto que englobe toda la dimensión en lo que a pobreza se refiere, es el punto de partida para el análisis de la investigación; es decir, la investigación tomará en cuenta el ingreso y su distribución para realizar las conclusiones pertinentes en el trabajo.

Según SEDESOL la pobreza "es la carencia de recursos necesarios para satisfacer la necesidad de una población o grupo de personas específicas, sin tampoco tener la capacidad y oportunidad de cómo producir esos recursos necesarios". La carencia de recursos estará medida por los ingresos y la población que se tomará en cuenta será la de Nicaragua. 


\section{Métodos de medición de desigualdad y pobreza}

El PIB per cápita: El PIB per cápita es el producto interno bruto dividido por la población a mitad de año. El PIB es la suma del valor agregado bruto de todos los productores residentes en la economía más todo impuesto a los productos, menos todo subsidio no incluido en el valor de los productos. Se calcula sin hacer deducciones por depreciación de bienes manufacturados o por agotamiento y degradación de recursos naturales. Los datos se expresan en dólares de los Estados Unidos a precios constantes (Banco Mundial, 2017).

$\boldsymbol{P I B}$ real: El producto interno bruto real es también conocido como PIB a precios constantes. Es el valor monetario de todos los bienes y servicios producidos por un país o una economía.Para calcular el mismo se deben considerar los precios del año que se toma como base. El objetivo de cálculo del PIB es obtener la producción de una economía y su evolución a lo largo del tiempo eliminando el efecto de la subida de precios o inflación.

Tasa de desempleo: El empleo genera ingresos que abaten directamente la pobreza; es decir, se debe considerar el supuesto de que la pobreza disminuye a tasas de desempleo relativamente bajas si se mantiene constante en el medio plazo. "El desempleo es la proporción de la población activa que no tiene trabajo pero que busca trabajo y está disponible para realizarlo (Banco Mundial, 2017).

Índice de Gini: Mide la desigualdad económica de una sociedad mediante la exploración del nivel de concentración que existe en la distribución de los ingresos entre la población. El coeficiente de Gini toma valores entre 0 y 1 ; un valor que tiende a 1 refleja mayor desigualdad en la distribución del ingreso. Por el contrario, si el valor tiende a cero, existen mayores condiciones de equidad en la distribución del ingreso (CONEVAL, 2017).

Brecha de pobreza: La brecha de la pobreza a 1,90 dólares al día (PPA 2011) es el déficit promedio de ingreso o consumo de la línea de pobreza de $\$$ 1.90 al día (que cuenta el no pobre como de cero), expresado como un porcentaje de la línea de pobreza. Esta medida refleja la profundidad de la misma , así como su incidencia (Banco Mundial, 2017).

Tasa de incidencia de la pobreza: Tasa de incidencia de la pobreza, sobre la base de la línea de pobreza nacional (\% de la población) (Banco Mundial, 2017).

\section{RESULTADOS Y DISCUSIÓN}

\section{Rasgos generales de la economía de Nicaragua}

La grafica siguiente muestra el comportamiento del PIB real nicaragüense en base a precios del 2010. Es evidente que en el periodo de 1980-1991 el país sufrió un grave retroceso en el ingreso nacional, aunque es preciso señalar que la tendencia en general a largo plazo en el período 19602015 ha sido creciente. 


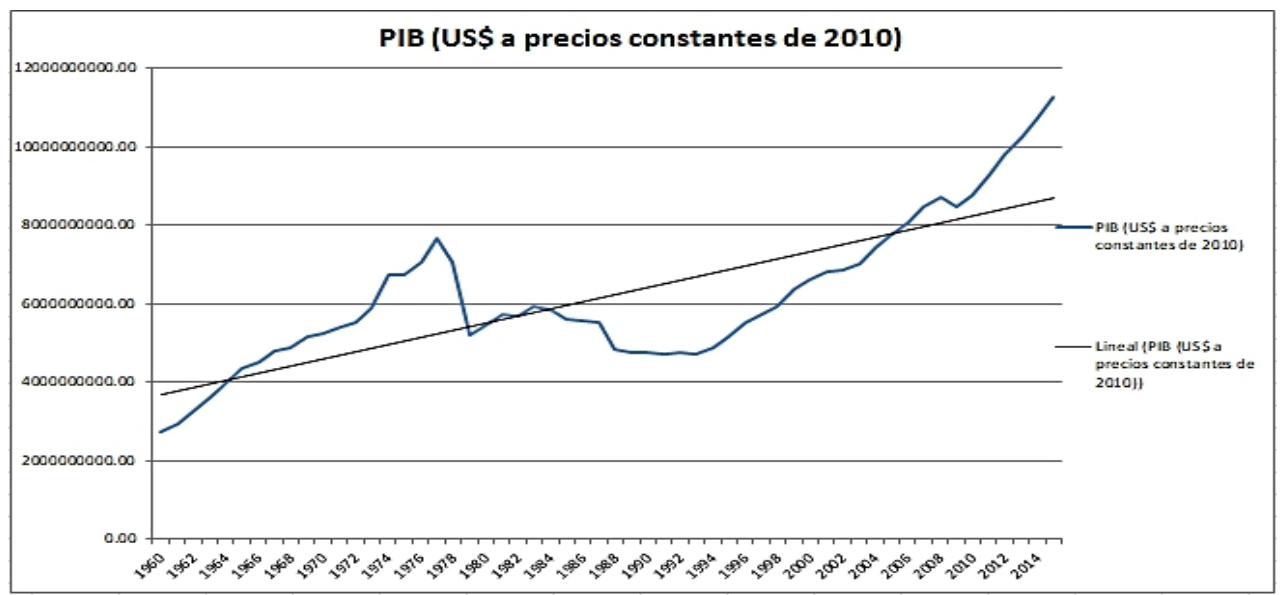

Figura 1. Fuente: Elaboración propia con datos del Banco Mundial

A partir de esta época se adoptaron las medidas sugeridas en el consenso de Washington, las cuales estaban enfocadas específicamente en la orientación de los gobiernos latinoamericanos. Dicho consenso proponía como principales medidas una reducción del gasto fiscal, a lo que Nicaragua respondió con una reducción de su ejército principalmente y privatizar las empresas estatales. Para llevar una "sana" macroeconomía se hicieron ajustes estructurales tales como la restructuración de la moneda, donde se cometió la grave aberración económica de situar el córdoba (moneda nacional) en paridad con el dólar. Estos mismos errores que se venían arrastrando desde 1985 con el plan Bertha (plan de ajuste estructural enfocado en abatir la inflación), lo que claramente afecta las exportaciones de un país.

A partir de 1990 con la entrada de las medidas adoptadas en Washington el país comenzó a recuperarse económicamente y es apreciable en la gráfica siguiente:

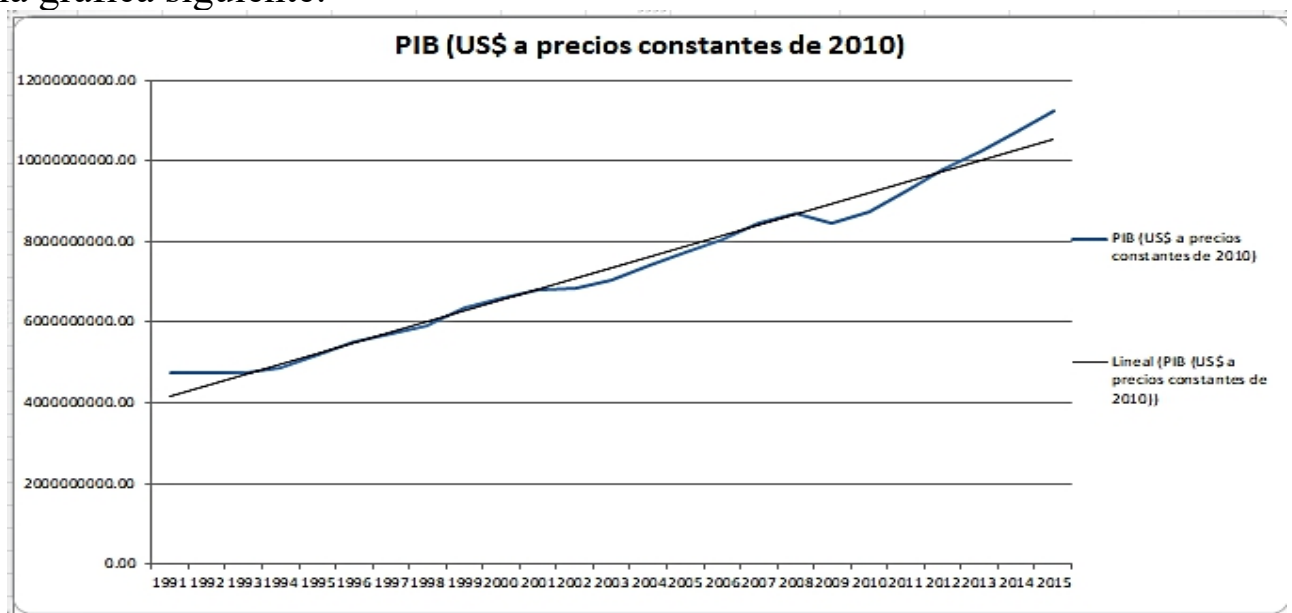

Figura 2. Fuente: Elaboración propia con datos del Banco Mundial. 


\section{Generación de ingresos (disminución de la pobreza) a través del empleo}

El trabajo es la fuente de generación de valor y a la vez de riqueza dentro del sistema. En el proceso de reproducción del capital, el capitalista se apropia de una parte de la riqueza generada por la fuerza de trabajo; esta es la manera de que el trabajador asalariado logre cubrir sus necesidades, a través del salario otorgado por su trabajo, que desde luego lo obtiene con la obtención de un empleo.

Entonces, al analizar la desigualdad es necesario también analizar el empleo porque abate directamente las desigualdades generadas por el sistema de reproducción capitalista cuya definición es: "El desempleo es la proporción de la población activa que no tiene trabajo pero que busca trabajo y está disponible para realizarlo (Banco Mundial, 2017)". Tomando esto en cuenta en la siguiente gráfica se muestra la tasa de desempleo de Nicaragua.

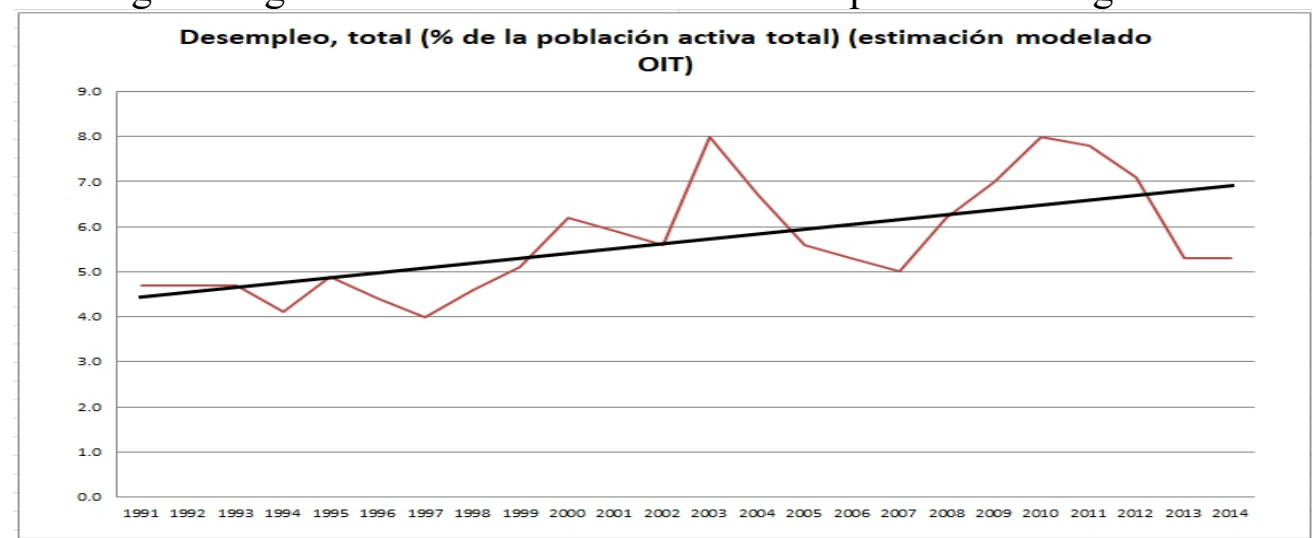

Figura 3. Fuente: Elaboración propia con datos del Banco Mundial.

A través de esta grafica se observa que, aunque en el país exista un crecimiento económico a partir de la introducción de las políticas neoliberales, el desempleo sigue teniendo una tendencia creciente. La teoría macroeconómica dicta que tasas sostenidas de crecimiento económico ayudarían a abatir el desempleo a mediano plazo, sin embargo en el período observado hay 24 años y aun así la tasa de desempleo permanece persistentemente.

Es más alarmante el hecho que la contabilización de los desempleados en Nicaragua no se realiza de manera correcta o conforme el empleo es necesario para salir de la pobreza y eliminar las desigualdades sociales que según (Peralta, 2010). "Trabajo productivo con remuneración justa; seguridad en el lugar de trabajo y protección social para las familias, mejores perspectivas para el desarrollo personal y la integración social" es la definición de la OIT de empleo decente, y es justamente este empleo el que ayudaría a reducir la desigualdad en Nicaragua. 
Marx publicó el primer tomo del Capital en 1867, enfrentándose a unas condiciones económicas, políticas y sociales diferentes a las actuales, razón por la cual resulta una dificultad al extrapolar directamente sus observaciones. En esa época los trabajadores industriales sufrían de miseria, vivían en tugurios urbanos, tenían largas jornadas de trabajo y el salario era muy bajo.

Es allí donde se comienza a generar la desigualdad, por la remuneración que obtienen aun los que tienen trabajo sin tomar en cuenta la tasa de desempleo en Nicaragua; como señala Piketty "el periodo de posguerra y auge económico de Estados Unidos hizo que comenzaran la medición de desigualdad"; Kuznets encontró que había una fuerte reducción de la desigualdad de los ingresos entre 1913 y 1948, período generalizado por la posguerra.

\section{Brecha de Pobreza y mediciones de la desigualdad}

El concepto de pobreza se ha tratado siempre desde una perspectiva de recursos disponibles, de renta disponible, de posesión o no posesión de ingresos para proveerse mínimamente de los recursos materiales suficientes y necesarios para la subsistencia y para una supervivencia digna. Transformándolo a la evolución de los sistemas económicos, es la privación involuntaria de todos aquellos recursos que impiden satisfacer; al menos, un mínimo de servicios de salud, nutrición y educación.

Se parte del hecho de que ser pobre no es una opción voluntaria; todo lo contrario, viene impuesta por las condiciones sociales, económicas, políticas y hasta culturales en las que se viven o se ha vivido; la sociedad moderna nos ha traído otra forma de comprender la privación y la pobreza.

No sería alejado de la realidad sostener esta misma hipótesis para Nicaragua debido a que en el período siguiente a los movimientos armadosestá demostrado que existe un crecimiento sostenido del producto interno bruto. El estadístico más indicado que se podría emplear para medir la desigualdad es el coeficiente de Gini.

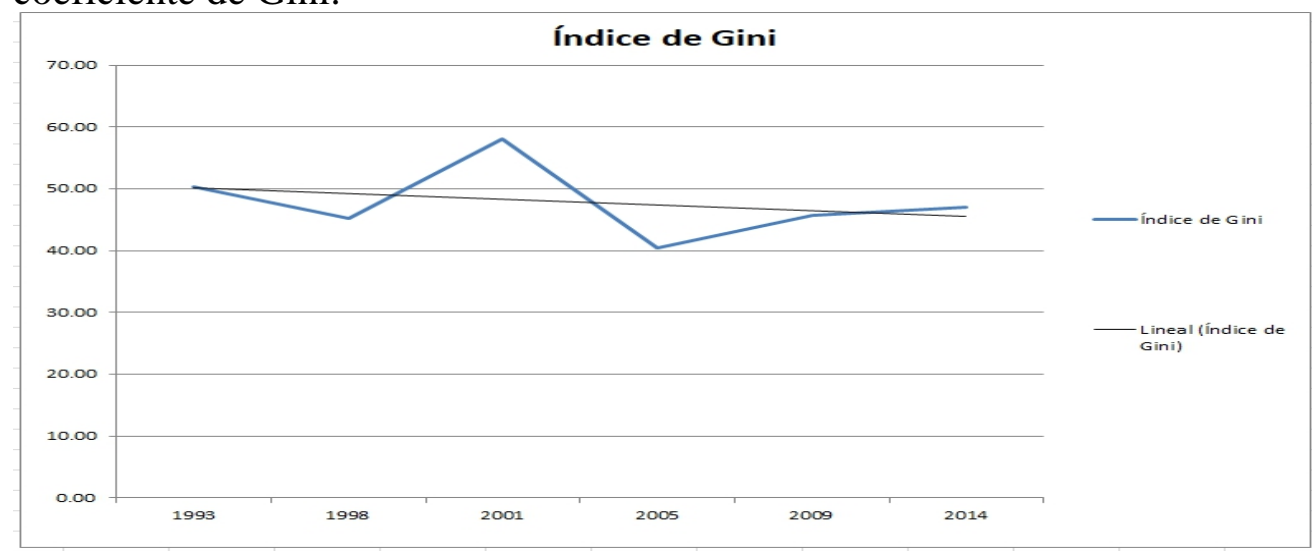

Figura 4. Fuente: Elaboración propia con datos del Banco Mundial. 
A pesar del número limitado de observaciones, la línea tendencial demuestra que la desigualdad en Nicaragua ha sido decreciente en el país y que poco a poco ha eliminado las desigualdades sociales.

A pesar de este análisis, es necesario indagar más en el tema; es por ello que debemos analizar aquello a lo que el Angus Deaton llama "gran escape". El autor analiza el bienestar que para él "se refiere a la satisfacción de las personas con su vida en general, sin perder de vista la felicidad, la salud y la economía, el aspecto psicológico, la educación y la participación social del individuo".

Es por ello que el análisis de desigualdad no se debe limitar solo al aspecto de ingresos y su distribución en Nicaragua. De lo anterior, el índice de Gini demuestra que la desigualdad de ingresos presenta una tendencia decreciente pero más allá aun en esta investigación. Como hizo Deaton, hay que ver cuántas personas han salido del umbral de la pobreza.

Siguiendo con los apartados aparece lo señalado anteriormente; la lógica macroeconómica indica que para reducir el desempleo es necesario mantener un crecimiento económico sostenido. Nicaragua ha mantenido un crecimiento económico sostenido en el período analizado (1991-2015), pero se puede observar que no existe dicha congruencia con la teoría macroeconómica. Sin embargo, a partir del año 2009, la tasa de desempleose ha visto particularmente reducida y entre 2013 y 2015 se mantuvo estable, cercana al $5 \%$, en proporción de la PEA. Esto significa que el ingreso disponible es más generalizado en la PEA y el consumo se incrementó debido al crecimiento constante de la PEA.

Un indicador de profundidad de la pobreza y su incidencia sobre la población es cómo mide el Banco Mundial a las poblaciones cuando no alcanzan a obtener $1.90 \$$ al día para poder acceder a la canasta bienes y servicios básicos que representa la línea de pobreza. En este caso, esto se denomina "brecha de pobreza.

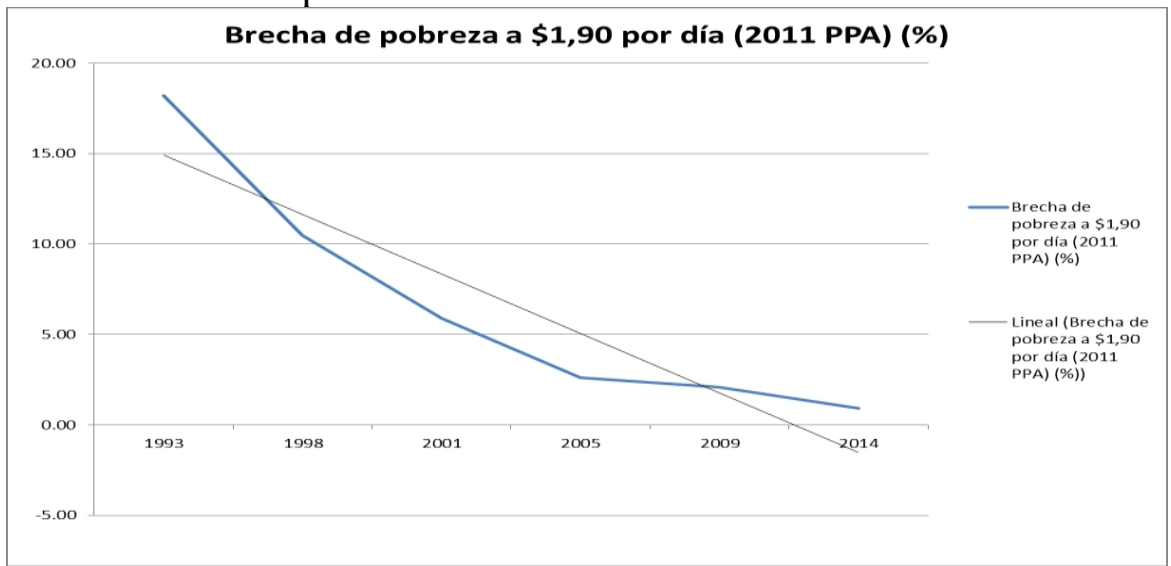

Figura 5. Fuente: Elaboración propia con datos del Banco Mundial. 
La brecha de pobreza anterior resulta un poco alentadora pues el porcentaje de la población que se encuentra dentro de esta brecha ha disminuido a un ritmo acelerado en el periodo de postguerra civil en Nicaragua. La línea tendencial, aunque imprecisa debido a insuficiencia de datos, principalmente se puede concluir que se encuentra cerca de llegar a cero, lo que significaría que todas las personas poseen los suficientes recursos para lograr alcanzar los bienes y servicios básicos que los coloquen arriba de la línea de pobreza.

Suponiendo que se puede seguir clasificando a un ser humano y dejemos de lejos los aspectos morales que implica, esto es, decir lo que se podría pensar realmente del consumo que se genera a partir de este miserable poder de compra con que cuentan las personas, dejaremos de lado el factor de nutrición y más aún el desarrollo de capacidades que plantea Sen, quien con lógica común determina con facilidad que no logra alcanzar el desarrollo de las capacidades y más aun no alcanzar el bienestar o siquiera una aproximación a lo que propone Deaton.

Es evidente que en esta brecha las personas que vivían con un ingreso o consumo con un déficit de $\$ 1.90$ por día han disminuido considerablemente. Está claro que la miseria en general ha disminuido. A pesar de que no se puede demostrar anualmente con una tasa de crecimiento debido a la insuficiencia de datos, los resultados a mediano plazo son notables; gracias a esto se puede concluir entonces que los pobres en general han disminuido. Otra medida a la cual el BM le llama "Tasa de incidencia de la pobreza, sobre la base de la línea de pobreza nacional (\% de la población) (Banco Mundial, 2017)"

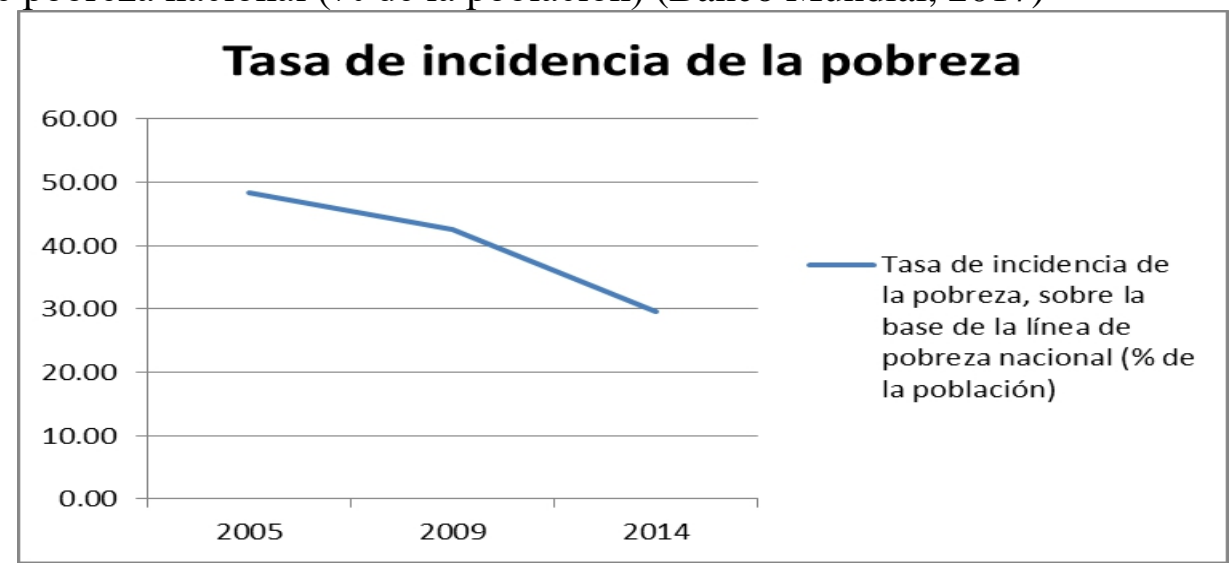

Figura 6. Fuente: Elaboración propia con datos del Banco Mundial.

En el año 2005, que es el primer dato emitido de este estadístico, el porcentaje de población pobre era de $48.3 \%$ en cuatro años; es decir, en 2009 la pobreza estuvo presente en el $42.5 \%$ de la población, lo cual implica que hubo una reducción de la incidencia de la pobreza en un 5.8\%. Entre las diferencias que se muestran en las observaciones que se hicieron en 2009 y 
2014 existe una diferencia mucho más amplia; 5 años después pasa de $45.5 \%$ a ser en 2014 de $29.6 \%$; hubo una reducción de la masa de pobres de $12.9 \%$ en 5 años y si se toma en cuenta la reducción de los 9 años en total, la pobreza se redujo en $18.7 \%$.

Se puede dar un acercamiento pero no una conclusión a partir de los datos de pobreza. Si se parte del supuesto de que la pobreza es un fenómeno involuntario a los agentes económicos racionales, las personas lograron salir de la pobreza por propia voluntad; pero él ¿por qué? es la pregunta, y en realidad es el hecho de que las personas ignorarían una pobreza absoluta si todos tuviesen los mismos bienes materiales. En realidad, la desigualdad o desequilibrio que tienen en lo que respect a los bienes que poseemos es la manera en que se dan cuenta que en realidad son pobres e inherentemente a la condición de ser un humano los impulsa a una igualdad, en este caso expresado por el hecho de buscar el mismo bienestar, expresado por los bienes materiales que otros individuos poseen.

\section{Encuesta Nacional de Hogares sobre Medición de Nivel de Vida 2014}

Al trabajar con la temática de desigualdad y pobreza se deben analizar también estadísticas del Instituto Nacional de Información de Desarrollo (INIDE) nicaragüense. En la revisión del sitio web se encuentra la "Encuesta Nacional de Hogares sobre Medición de Nivel de Vida 2014 (INIDE, 2016)" en su versión de informe. Por lo tanto, se analizará a continuación lo más contundente del informe en lo que se refiere a pobreza, la distribución de la misma y el bienestar medido por los ingresos de los encuestados.

El primer elemento destacable de la encuesta es bienestar medido mediante el consumo, el cual es una manera ingeniosa de aproximar el ingreso. Partimos del hecho de que el consumo depende de la renta actual como indica Keynes, quien afirma que el ahorro no influye en el consumo. Este supuesto se asume a través de la premisa de que los pobres no ahorran y su consumo principalmente sólo depende de la renta actual y no de una riqueza obtenida con anterioridad. La variación del bienestar medido mediante el consumo de las familias encuestadas está representada por "El gasto anual per cápita en el período 2009 - 2014 creció significativamente a nivel nacional en $33.7 \%$ (INIDE, 2016).

De igual forma fueron analizadas dos líneas de pobreza, una de pobreza general y una de personas con pobreza moderada. "Los valores monetarios de las Líneas de Pobreza Extrema y General en Córdobas reales se incrementaron en $8.8 \%$ y $3.6 \%$ respectivamente entre los años 2009 y 2014 (INIDE, 2016).

En materia de distribución de la pobreza, se encontró que "La pobreza general y pobreza extrema en Nicaragua es mayoritariamente rural. Los datos muestran que para el 2014 , del $100 \%$ de los pobres generales del país, el $71 \%$ provienen del área rural mientras que $29 \%$ residen en el área urbana. Una situación análoga se presenta con la pobreza extrema: el 83\% de los pobres 
extremos del país residen en el área rural y el 17\% en el área urbana. La proporción de población por debajo de la línea de pobreza general se redujo en 12.9 puntos porcentuales, pasando de $42.5 \%$ a $29.6 \%$ entre 2009 y 2014 . En cuanto a la pobreza extrema, se ha reducido en 6.3 puntos porcentuales entre esos cinco años (INIDE, 2016).

Con respecto a la desigualdad "A nivel nacional, el 20\% más rico de la población acumula el $45.4 \%$ del gasto total (gasto promedio anual per cápita de C\$71,861.4), mientras que el 20\% más pobre acumula el 6.8\% (gasto promedio anual per cápita de $\mathrm{C} \$ 10,772.3)$. Esto significa que el gasto promedio anual per cápita de los más pobres es 6.5 veces menor que el quintil más rico. De manera semejante, el $40 \%$ más rico de la distribución, acumula dos tercios del total del gasto y los quintiles restantes, acumulan únicamente un tercio del mismo (INIDE, 2016).

Existe una diferencia abismal entre el consumo de los más pobres y los más ricos. Como se señaló, es una diferencia de 6.5 veces para ser más precisos. Se calculó el índice de Gini como medida de distribución del ingreso nacional. El coeficiente de Gini rindica un valor de 0.48 puntos a nivel nacional. En términos comparativos, dicho índice tuvo un leve incremento de 2 puntos, en el periodo 2009 -2014 evolucionando de 0.46 a 0.48 .

En el área urbana el incremento fue de 4 puntos y en la zona rural la desigualdad se mantuvo estable (INIDE, 2016)". Estos resultados son principalmente congruentes con la teoría Marxista sobre la reproducción y acumulación del capital, porque radican principalmente en el hecho contundente de que la reproducción del capital se da principalmente en la zona urbana. Las actividades económicas se dan principalmente en la zona urbana y es aquí donde se confirma debido a que en esta área urbana es que se da el incremento del índice de Gini. Es aquí donde está la principal actividad de reproducción del capital donde $y$ se generan las desigualdades independientemente de otros fenómenos implícitos que ayuden a la desigualdad social.

\section{Conclusion}

La primera conclusión y quizás la más difícil de admitir es que en realidad, a pesar del trabajo realizado, a través del análisis de estadísticas frías “jamás se logrará transmitir lo que en realidad significan estos desequilibrios. No hay una total consciencia del sufrimiento que causan los rezagos de este modo de producción.

La segunda está intrínseca en la definición de bienestar de Deaton. En realidad, la desigualdad en la investigación se valoró de la manera clásica, es decir, simplemente medido por el ingreso y un índice de concentración muy célebre como lo es el de Gini. Sin embargo, al observar más allá, debería medir las desigualdades de una forma multidimensional para detectar los principales 
factores que siguen fomentando la desigualdad y combatirlos más severamente.

Concretamente para Nicaragua se analizó el periodo a partir de 1991 por el hecho de que fue el año en el que se integraron las políticas neoliberales en el país. A pesar de los logros reconocidos por las organizaciones internacionales (UNESCO) en materia de salud y educación en la década de los $80 \mathrm{~s}$, es innegable que el periíodo de guerra deterioró las capacidades productivas del país; es por ello que se analiza el periodo de la senda de crecimiento que es donde se supone se generó crecimiento económico y aumento en el empleo, se pueden reducir las desigualdades.

Se constató que la pobreza ha disminuido drásticamente en el periodo analizado; ahora en la tasa general de incidencia de la pobreza se disminuyó hasta menos del 30\% de la población en 2014; cuando en 2005 casi el 50\% de la población nicaragüense era pobre. También es destacable que los pobres que viven en la brecha de $1.90 \$$ al día ahora son menos. La reducción de la pobreza extrema y moderada aportada por el Coeficiente de Gini que mide la desigualdad, en el periodo 2009 -2014 evolucionó de 0.46 a 0.48 .

En el área urbana el incremento fue de 4 puntos y en la zona rural la desigualdad se mantuvo estable (INIDE, 2016)". Estos resultados son principalmente congruentes con la teoría Marxista sobre la reproducción y acumulación del capital, porque radica principalmente en el hecho contundente de que la reproducción del capital se da principalmente en la zona urbana. Cuando comenzó la industrialización inglesa, período en el que Marx desarrolló su tesis, este señaló que el proceso capitalista se da en Londres, la principal urbe de su época; al igual que en ese tiempo el progreso económico se da principalmente en la zona urbana. Es aquí donde se confirma la teoría debido a que en esta área urbana es que se da el incremento del índice de Gini; en otras palabras es aquí donde está la principal actividad de reproducción del capital y donde se generan las desigualdades independientemente de otros fenómenos implícitos que ayuden a la desigualdad social.

\section{References:}

1. Banco Mundial (2017). Banco Mundial. [En línea]. Available at: http://datos.bancomundial.org/indicador/SL.UEM.TOTL.ZS [Último acceso: 28112017$]$.

2. CONEVAL (2017). CONEVAL. [En línea]. Available at: http://www.coneval.org.mx/Medicion/Paginas/Cohesion_Social.aspx [Último acceso: 29112017 ].

3. Cortés, M. M. S. J. P. P. S. F. (2018). Biblioteca Virtual Clacso. [En línea]. http://bibliotecavirtual.clacso.org.ar/ar/libros/costar/flacso/cuad131.pd $\mathrm{f}$ 
4. INIDE (2016). Instituto Nacional deInformación de Desarrollo. [En línea]. Available at: http://www.inide.gob.ni/Emnv/Emnv14/EMNV\%2020142\%20Febrero\%202016.pdf [Último acceso: 3011 2016].

5. Ortiz, L. P. (2009). Universidad Autónoma de Madrid. [En línea]. Available at: https://www.uam.es/personal_pdi/economicas/laurap/EEM/3Desigualdad.pdf [Último acceso: 201611 29].

6. Pampillón, R. (2009). Economy weblog. [En línea]. Available at: http://economy.blogs.ie.edu/archives/2009/10/\%C2\%BFque-es-elindice-de-desarrollo-humano-idh.php [Último acceso: 2811 2016].

7. Peralta, E. (2010). Perspectiva Laboral en México. comercio exterior, pp. 195-208.

8. Sen Amartya, K. (1992). Inequality Reexamined. Cambridge: Harvard University, 0101 , pp. -

9. Torres, A. (2017). Debate. [En línea]. Available at: https://www.debate.com.mx/mexico/La-desigualdad-tiene-su-ladopositivo-Deaton-20170922-0276.html 